\title{
Approach to geriatric patients with subclinical thyroid disorders
}

\author{
Graziano Ceresini $\cdot$ Michela Marina
}

Received: 30 May 2014/ Accepted: 11 June 2014/Published online: 4 July 2014

(C) Springer Science+Business Media New York 2014

Changes in circulating concentrations of free thyroxine (FT4), free triiodothyronine (FT3) and thyrotropin (TSH) participate in the overall readjustment of the hormonal status that occur with aging $[1,2]$. While this phenomenon may be considered a characteristic of the aging processes, there is no doubt that well-defined thyroid dysfunctions, especially of subclinical type, are more frequently observed in aging individuals compared to young-adult subjects. This scenario may represent an important point in order to correctly assess elderly subjects in relation to their thyroid status.

In this issue of Endocrine, e Silva et al. [3] evaluated the prevalence of thyroid dysfunction in elderly subjects attending an outpatient clinic and assessed whether subclinical hypothyroidism (SHypo) affected functional status, which was evaluated by the well-known scores for activities of daily living (ADLs) and instrumental activities of daily living (IADLs), cognitive performance or depressive symptoms. Cognitive impairment or depressive symptoms did not differ among thyroid status groups, regardless of age. However, regarding the functional status, the authors found that while SHypo did not reduce performance in either ADLs or IADLs in elderly subjects as a whole, it was an independent protective factor against dependence in ADLs and IADLs in subjects aged 85 years or older. The conclusion of this study is that serum TSH above the reference may be associated with better functional status in subjects aged 85 years or older.

G. Ceresini $(\square) \cdot$ M. Marina

Department of Clinical and Experimental Medicine,

Endocrinology of Aging Unit, University of Parma, University

Hospital of Parma, Via Gramsci 14, 43126 Parma, Italy

e-mail: ceresini@unipr.it
The relationship between functional capacity and thyroid status is a matter of debate. For example, in a recent report, Virgini et al. [4] reported no substantial differences in functional capacity between either SHypo or subclinical hyperthyroidism (SHyper) and euthyroidism in elderly subjects, although in their study the age of the subjects was lower than that reported in the study by e Silva et al. However, the critical evaluation of the results reported by e Silva et al. [3] must take into account that the number of subjects in this study was quite low (i.e., 43 SHypo subjects were compared with 235 euthyroid subjects), although this is a characteristic shared by many studies which have dealt with aging and thyroid dysfunctions. Another point to be underlined is represented by the fact that in the study by e Silva et al. circulating TSH levels which defined Shypo were modestly elevated (i.e., $5.5 \pm 1.2$ [Mean $\pm \mathrm{SD}$ ] $\mathrm{mIU} / \mathrm{L}$ ). Although the levels of circulating TSH is not always reported in the literature when defining groups of subjects affected by SHypo, this point may be of importance. For example Rodondi et al. [5], in a cohort of elderly subjects affected by SHypo, demonstrated that, compared with euthyroid participants, congestive heart failure events occurred more frequently among those with a TSH level of $7.0 \mathrm{mIU} / \mathrm{L}$ or greater but not among those with TSH levels between $4.5-6.9 \mathrm{mIU} / \mathrm{L}$.

Therefore, questions could be raise on whether the protective effects of SHypo on functional activities, claimed by e Silva et al., would be still observed in SHypo subject with circulating TSH concentrations higher than those reported in their subjects.

Nevertheless, the point raised by the authors underline an important question. Is there the possibility that, in the oldest-old, the clinical relevance of SHypo has to be considered in a different way in comparison with young-adult or middle-aged subjects? This concept has been already 
hypothesized by literature reports. For example, in an extensive review by Biondi and Cooper [6] it was underlined that, according to epidemiological studies, the risk of coronary heart disease is increased in young and middleaged patients, but not in elderly patients, with SHypo and that SHypo appeared to exert a protective cardiovascular effect in patients older than 85 years. In line with these observations, more recent reports have demonstrated that the negative impact of SHypo on cardiovascular risk, which is recognized in young adults, is less evident in elderly subjects aged 85 years or older [7].

All these reports would suggest that the management of elderly SHypo patients should be age-dependent. In moderately old patients it could be similar to that followed for adults, probably including the possibility of treatment options, whereas the oldest-old could be managed, in most cases, with a wait-and-see strategy before hormonal treatment.

The perspective could be different for aging patients affected by SHyper, a thyroid dysfunction which may reach an important prevalence in iodine-deficient areas [8]. Although with some contrasting reports, literature data have demonstrated that SHyper may be associated with several negative clinical outcomes, including abnormalities of the cardiovascular system, bone metabolism, blood coagulation, cognition, and increased mortality [9].

It is particularly important to consider that the presence of signs and symptoms of hyperthyroidism are often masked in the elderly even in patients with severe overt disease: this phenomenon, together with the fact that the clinical consequences of SHyper are potentially more significant in the elderly compared with younger subjects [6], must be taken into account when evaluating elderly individuals.

Treatment of SHyper is still a matter of debate, since no controlled intervention studies to provide evidences of benefits have been performed. However, with this limitation being recognized, the guidelines on the treatment of thyrotoxicosis, released by the American Thyroid Association in conjunction with the American Association of Clinical Endocrinologists, in 2011 [10], report that treatment of SHyper should be considered in all individuals aged 65 years or older in whom TSH is persistently $<0.1$ $\mathrm{mIU} / \mathrm{L}$.

Therefore, both diagnosis and treatment of subclinical thyroid dysfunctions represent a clinical challenge in elderly subjects and differences between observational studies in number of subjects, levels of circulating TSH, age of the subjects, iodine content of the geographic area, and genetic of populations have to be taken into account when evaluating results. Although more defined conclusions about the need of treatment of elderly-associated thyroid dysfunctions could only derive from sufficiently large, long-term, randomized controlled trials, accurate examination of elderly patients with suspicion of thyroid dysfunction, including the complete evaluation of the clinical context, is mandatory in order to individualize the appropriate clinical management.

Conflict of interest The authors have no conflict of interest to disclose.

\section{References}

1. S. Mariotti, C. Franceschi, A. Costarizza, A. Pinchera, The aging thyroid. Endocr. Rev. 16, 686-715 (1995)

2. M.I. Surks, J.G. Holowell, Age-specific distribution of serum thyrotropin and antithyroid antibodies in the US population: Implication for the prevalence of subclinical hypothyroidism. J. Clin. Endocrinol. Metab. 92, 4575-4582 (2007)

3. S.O. e Silva, I.T. Chan, M.A. Lobo Santos, M. Cohen, P. de La Roque, M. Araujo, J. da Silva Almeida, A. Simões, H.R.B. Givigi, M. Vaisman, C.M. Paixao Jr, S.P. de Fatima dos Teixeira, Impact of thyroid status and age on comprehensive geriatric assessment. Endocrine. (2013). doi:10.1007/s12020-013-0077-x

4. V.S. Virgini, L.W. Wijsman, N. Rodondi, D.C. Bauer, P.M. Kearney, J. Gussekloo, W.P.J. den Elzen, J.W. Jukema, R.G.J. Westendorp, I. Ford, D.J. Stott, S.P. Mooijaart, on behalf of the PROSPER Study Group, Subclinical thyroid dysfunction and functional capacity among elderly. Thyroid. 24, 208-214 (2014)

5. N. Rodondi, A.B. Newman, E. Vittinghoff, N. de Rekeneire, S. Satterfield, T.B. Harris, D.C. Bauer, Subclinical hypothyroidism and the risk of heart failure, other cardiovascular events, and death. Arch. Intern. Med. 165, 2460-2466 (2005)

6. B. Biondi, D.S. Cooper, The clinical significance of subclinical thyroid dysfunction. Endocr. Rev. 29, 76-131 (2008)

7. G. Pasqualetti, S. Tognini, A. Polini, N. Caraccio, F. Monzani, Is subclinical hypothyroidism a cardiovascular risk factor in the elderly? J. Clin. Endocrinol. Metab. 98, 2256-2266 (2013)

8. G. Ceresini, F. Lauretani, M. Maggio, G.P. Ceda, S. Morganti, E. Usberti, C. Chezzi, R. Valcavi, S. Bandinelli, J.M. Guralnik, A.R. Cappola, G. Valenti, L. Ferrucci, Thyroid function abnormalities and cognitive impairment in elderly people: Results of the invecchiare in chianti study. J. Am. Geriatr. Soc. 57, 89-93 (2009)

9. G. Ceresini, G.P. Ceda, F. Lauretani, M. Maggio, E. Usberti, M. Marina, S. Bandinelli, J.M. Guralnik, G. Valenti, L. Ferrucci, Thyroid status and 6-year mortality in elderly people living in a mildly iodine-deficient area: The aging in the chianti area study. J. Am. Geriatr. Soc. 61, 868-874 (2013)

10. R.S. Bahn, H.B. Burch, D.S. Cooper, J.R. Garber, M.C. Greenlee, I. Klein, P. Laurberg, I.R. McDougall, V.M. Montori, S.A. Rivkees, D.S. Ross, J.A. Sosa, M.N. Stan, Hyperthyroidism and other causes of thyrotoxicosis: Management Guidelines of the American Thyroid Association and American Association of Clinical Endocrinologists. Thyroid. 21, 593-646 (2011) 